ARTICLE

\title{
Selectively tuning ionic thermopower in all-solid- state flexible polymer composites for thermal sensing
}

\author{
Cheng Chi ${ }^{1,6}$, Meng An (10) 1,2,6, Xin Qi ${ }^{1}$, Yang Li ${ }^{3}$, Ruihan Zhang ${ }^{4}$, Gongze Liu ${ }^{3}$, Chongjia Lin (1) ${ }^{3}$, He Huang ${ }^{3}$,

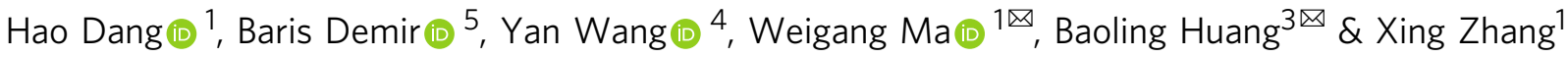

There has been increasing interest in the emerging ionic thermoelectric materials with huge ionic thermopower. However, it's challenging to selectively tune the thermopower of all-solidstate polymer materials because the transportation of ions in all-solid-state polymers is much more complex than those of liquid-dominated gels. Herein, this work provides all-solid-state polymer materials with a wide tunable thermopower range $\left(+20 \sim-6 \mathrm{mV} \mathrm{K}^{-1}\right)$, which is different from previously reported gels. Moreover, the mechanism of $p$-n conversion in allsolid-state ionic thermoelectric polymer material at the atomic scale was presented based on the analysis of Eastman entropy changes by molecular dynamics simulation, which provides a general strategy for tuning ionic thermopower and is beneficial to understand the fundamental mechanism of the $p$ - $n$ conversion. Furthermore, a self-powered ionic thermoelectric thermal sensor fabricated by the developed $p$ - and $n$-type polymers demonstrated high sensitivity and durability, extending the application of ionic thermoelectric materials.

\footnotetext{
${ }^{1}$ Key Laboratory for Thermal Science and Power Engineering of Ministry of Education, Department of Engineering Mechanics, Tsinghua University, Beijing 100084, China. ${ }^{2}$ College of Mechanical \& Electrical Engineering, Shaanxi University of Science and Technology, Xi'an 710021, China. ${ }^{3}$ Department of Mechanical and Aerospace Engineering, The Hong Kong University of Science and Technology, Clear Water Bay, Hong Kong SAR, China. ${ }^{4}$ Department of Mechanical Engineering, Worcester Polytechnic Institute, 100 Institute Road, Worcester, MA 01609, USA. ${ }^{5}$ Centre for Theoretical and Computational Molecular Science, The Australian Institute for Bioengineering and Nanotechnology, The University of Queensland, Brisbane, QLD 4072, Australia. ${ }^{6}$ These

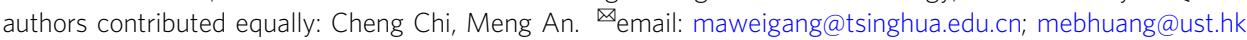


T hermal sensors (TS) play a crucial role in various areas such as aerospace, industry, agriculture, biomedical sensing, monitoring of microprocessors, and environmental temperature measurements ${ }^{1,2}$. Specifically, TS for heat detection must be accurate, efficient, scalable, and cost-effective. Thermoelectric (TE) technology is a powerful technology that can directly convert the temperature differences to the electric voltage based on the Seebeck effect with a lower level of vibration and noise, less maintenance necessities, precise temperature control and high reliability ${ }^{3-5}$. The traditional TE materials based on inorganic semiconductors $^{5}$ and semi-metals ${ }^{6,7}$ have been extensively investigated, however, they still suffer from low thermopower $\left(\sim 100-200 \mu \mathrm{V} \mathrm{K}^{-1}\right)$, less flexibility, low Earth abundance and substantial production cost which usually requires the integration of thousands of thermoelements connected in series, rendering them not conducive to large-scale industrial applications ${ }^{8-10}$.

Alternatively, high ionic thermopower or Seebeck coefficient $\left(S_{i}\right)$ was found in the ionic thermoelectric ( $i-\mathrm{TE})$ materials based on the Soret effect, providing a promising route to develop highperformance TE materials ${ }^{11-13}$. For example, polyethylene oxide $(\mathrm{PEO}) / \mathrm{NaOH} / \mathrm{H}_{2} \mathrm{O}$ solution ${ }^{14}$ exhibited a thermopower of $10 \mathrm{mV} \mathrm{K}^{-1}$, cellulose-polystyrene sulfonate sodium (NFC-PSSNa) had a thermopower of $8.4 \mathrm{mV} \mathrm{K}^{-1}$ at $100 \%$ relative humidity $(\mathrm{RH})^{15}$ and a synergistic $\mathrm{KCl} /$ ferro/ferricyanide $\left(\mathrm{FeCN}_{6}{ }^{4-/ 3-}\right)$ gelatin offered a high thermopower of $17.0 \mathrm{mV} \mathrm{K}^{-1}$, which is much higher than pure solutions of $\mathrm{KCl}\left(0.04 \mathrm{mV} \mathrm{K}^{-1}\right)$ and $\mathrm{FeCN}_{6}{ }^{4-/ 3-}$ $\left(1.4 \mathrm{mV} \mathrm{K}^{-1}\right)^{16}$. These liquid-dominated gels are found to exhibit larger thermopower than that of the liquid solution alone. In addition to the thermal transport difference between cations and anions, it is suspected that the enhancement on thermopower is also attributed to the Eastman entropy which was induced by more complex interatomic interactions among ions, polymers and surrounding media ${ }^{8,16}$. However, the quantitative relationship between complex interactions and the Eastman entropy change of cations and anions in all-solid-state polymer $i$-TE materials has not been demonstrated at the atomic scale to this end.

Moreover, as most polymer-based $i$-TE materials show positive thermopowers, several efforts have been attempted to investigate the $p$ - $n$ conversion in liquid $i$-TE materials, i.e 1-ethyl-3methylimidazolium acetate (EMIm:Ac) $/ \mathrm{H}_{2} \mathrm{O}$ solution ${ }^{17}$, iodide/ triiodide $\left(\mathrm{I}^{-} / \mathrm{I}_{3}{ }^{-}\right)$thermogalvanic cell ${ }^{18}$ liquid systems and 1ethyl-3-methylimidazolium bis(trifluoromethylsulfonyl)imide (EMIM:TFSI) based liquid-dominated gels ${ }^{9}$. However, few reports have reported the $p-n$ conversion in the all-solid-state $i$ TE polymer materials so far. Particularly, it's very significant to convert $p$-type all-solid-state $i$-TE material to $n$-type when fabricating high-performance $i$-TE modules. Compared with liquids and liquid-dominated gels, all-solid-state $i$-TE polymer materials provide unique merits, such as no leakage, high mechanical strength, ease in fabrication, and good durability. In addition, it's almost impossible to deposit a uniform metal contact layer on these liquids and gels as the liquid will be evaporated under a high vacuum or the liquid could result in a discontinuous contact layer due to rapid cooling, which seriously limited the practical applications. Despite the benefits of all-solid-state $i$-TE materials, the lacking of efficient negative thermopower still hindered the development of all-solid-state $i$-TE based devices ${ }^{9,19}$. It is very challenging to selectively tune the positive thermopower to negative of all-solid-state $i$-TE polymer materials because allsolid-state polymer systems are much more complex than those liquids and their performances are affected by various factors. Especially, the transportation of ions in the all-solid-state polymer is fundamentally different from that in liquid-dominated gels or liquids, in which the movement of ions relies on the interaction with the polar group of polymers combining with the segmental motion of polymer chains instead of the straightforward diffusion in the liquid-dominated gels ${ }^{20,21}$. Unluckily, fundamental studies into the mechanism of ionic thermopower conversion in all-solidstate polymers are lacking, and there is rarely a universal strategy reported to convert $p$-type thermopower to $n$-type, seriously impeding the fundamental understanding and the practical application of $i$-TE polymer materials-based devices.

To address these issues, our work successfully achieved the conversion from $p$-type all-solid-state polyvinylidene fluoridehexafluoropropylene (PVDF-HFP)/sodium bis(trifluorome thylsulfonyl)imide (NaTFSI)/propylene carbonate (PC) [PVDF-HFP/NaTFSI/PC (PhNP)] to $n$-type with a wide tunable ionic thermopower range $\left(+20\right.$ to $\left.-6 \mathrm{mV} \mathrm{K}^{-1}\right)$ by regulation of the transportation of $\mathrm{Na}^{+}$ions. The mechanism of the $p-n$ conversion based on analysis of Eastman entropy change in all-solid-state $i$-TE polymer material was presented at the atomic level. The molecular dynamics (MD) simulation results found that the conversion in thermopower is strongly related to Eastman entropy change in the charge type of the ions, as the entropy change of $\mathrm{Na}^{+}$cations is higher than that of TFSI ${ }^{-}$anions in the $p$-type PhNPs and vice versa, which provides a general strategy for tuning ionic thermopower in all-solid-state polymers. Besides a high-performance planar $i$ TE generator ( $i$-TEG), we further demonstrated an $i$-TE TS with high sensitivity, extending the application of $i$-TE materials.

\section{Results}

Thermoelectric properties of $p$-type PhNPs. The developed flexible all-solid-state $p$-type $i$-TE PhNP composites (Supplementary Fig. 1) are composed of PVDF-HFP, NaTFSI salt and low-molecular-weight PC, in which the weight ratios of NaTFSI/ PC to overall composite range from 30 to $86 \mathrm{wt} . \%$, which are assigned as PhNP-30 to PhNP-86, respectively and the preparation procedures are illustrated in Supplementary Information. The ionic thermopower was measured through a home-made inplane setup (Supplementary Fig. 2) which was calibrated with the reported materials in the previous reports ${ }^{8,9,14}$. To be noticed, the starting hot and cool ends of PhNPs were electrically connected to the positive and negative poles of a voltmeter, respectively. When a temperature difference $(\Delta T=+6 \mathrm{~K})$ was applied, a negative thermal voltage of PhNP-86 was produced (Fig. 1b). Once alternating of the hot and cool sides $(\Delta T=-6 \mathrm{~K})$, the generated TE voltage instantly turned to the positive direction correspondingly, demonstrating rapid and reversible thermal response behavior. The observations suggested a higher concentration of positive $\mathrm{Na}^{+}$cations accumulated at the cool side as illustrated in Fig. 1a. It is also implied the thermal mobility of $\mathrm{Na}^{+}$ions was larger than that of TFSI ${ }^{-}$anions, belonging to a $p$ type $i$-TE material ${ }^{9,16}$. Besides, the higher the temperature difference, the larger thermal voltage was generated by PhNPs, exhibiting high capability in thermal-intensity sensing. The $S_{i}$ of each PhNPs was obtained by fitting the slope of the measured $\Delta V_{i}-\Delta T$ curves (Supplementary Fig. 3 ) according to Eq. (1)

$$
S_{i}=\frac{V\left(T_{H}\right)-\left(T_{C}\right)}{T_{H}-T_{C}}
$$

where $V\left(T_{\mathrm{H}}\right)$ and $V\left(T_{\mathrm{C}}\right)$ correspond to the voltage of the hot electrode at temperature $T_{\mathrm{H}}$ and the cold electrode at temperature $T_{\mathrm{C}}$, respectively.

Interestingly, the thermopower of the PhNPs gradually increased with the increasing weight ratio of NaTFSI/PC and reached around $20 \pm 4 \mathrm{mV} \mathrm{K}^{-1}$ of PhNP-86 (Fig. 1c) at $68 \% \mathrm{RH}$, demonstrating high performance compared to the recently reported works, such as $11 \mathrm{mV} \mathrm{K}-1$ of $\mathrm{PEO} / \mathrm{NaOH} / \mathrm{H}_{2} \mathrm{O}^{14}$ and $13 \mathrm{mV} \mathrm{K}^{-1}$ of PVDF-HFP/EMIM:TFSI/polyethylene glycol 
a

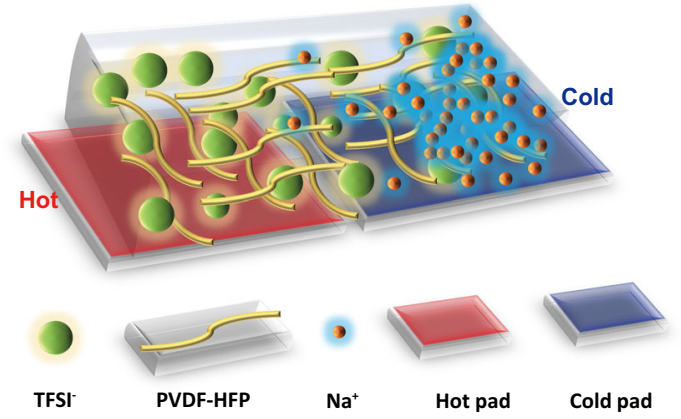

c

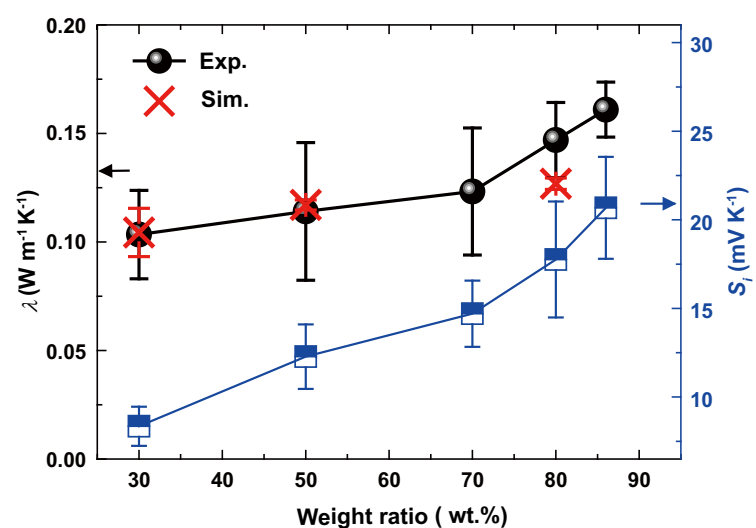

b

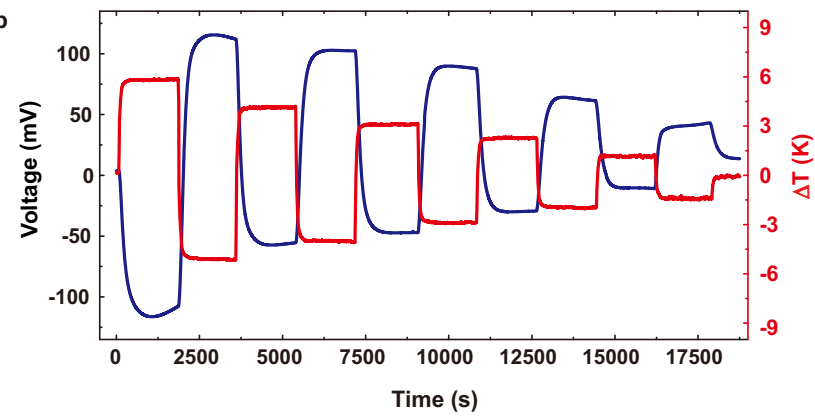

d

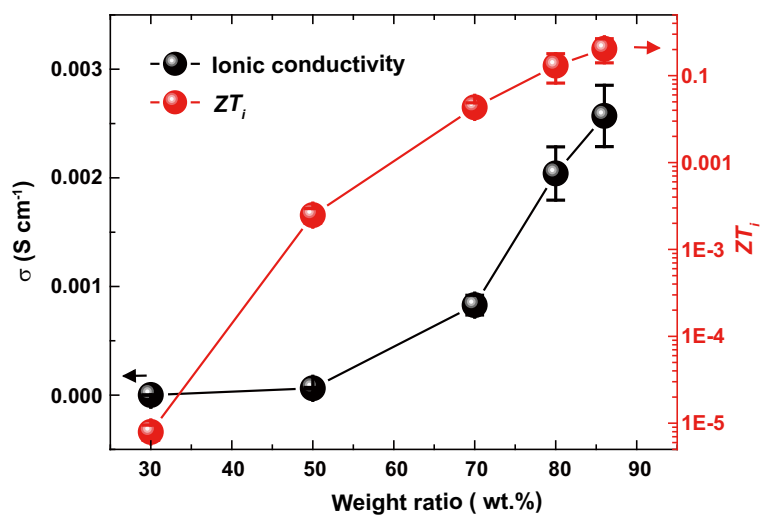

Fig. 1 The thermoelectric performance of the $\boldsymbol{p}$-type PhNP. a The diagrammatic illustration of the $p$-type (Na ${ }^{+}$cations dominate thermodiffusion) PhNP $i$-TE materials. b The plot of the measured $\Delta V_{i}-\Delta T$ curves of PhNP-86. c The measured thermopower, thermal conductivity together with simulation results of PhNPs. d The measured ionic conductivity and figure of merit $Z T_{i}$ of each PhNPs.

$(\mathrm{PEG})^{9}$. The ionic conductivity $\left(\sigma_{i}\right)$ of each PhNPs was measured by the electrochemical impedance method ${ }^{22}$ and the corresponding measured impedance spectra were plotted in Supplementary Fig. 4. Similarly, the ionic conductivity was improved from the PhNP-30 of $\sim 4 \times 10^{-7} \mathrm{~S} \mathrm{~cm}^{-1}$ to the highest value in PhNP-86 of $\sim 2.6 \times 10^{-3} \mathrm{~S} \mathrm{~cm}^{-1}$ (Fig. 1d) which is near to that of the pure liquid NaTFSI/PC $\left(6 \times 10^{-3} \mathrm{~S} \mathrm{~cm}^{-1}\right)$. At the high weight ratio of NaTFSI/PC, the PhNP-86 formed a relatively homogeneous and cross-linked porous structure as observed from the cross-section view in Supplementary Fig. 5, which can improve the diffusion coefficient of ions and facilitate the ions transport ${ }^{23,24}$. In addition, the thermal conductivity $\left(\lambda_{i}\right)$ of the PhNPs was measured through the Hot-disk method (Supplementary Fig. 6) and it exhibited an increasing trend from 0.1 to $0.16 \mathrm{~W} \mathrm{~m}^{-1} \mathrm{~K}^{-1}$ with increasing the weight ratio of NaTFSI/PC. The calculated thermal conductivities from MD simulations for PhNP-30, -50, -80 were around $0.1,0.117$ and $0.127 \mathrm{~W} \mathrm{~m}^{-1} \mathrm{~K}^{-1}$, respectively, which matched well with experimental data (Fig. 1c) and were consistent with the thermal conductivity range $\left(0.1-0.3 \mathrm{~W} \mathrm{~m}^{-1} \mathrm{~K}^{-1}\right)$ of amorphous materials ${ }^{25,26}$. Moreover, the TE performance of PhNP-86 with different concentrations of NaTFSI $(0.2,0.5,1.0$, and $2.0 \mathrm{M}$ ) was also studied, where the weight ratio of NaTFSI/PC to the overall PVDF-HFP/NaTFSI/PC was kept as $86 \mathrm{wt} . \%$. Both the ionic thermopower and conductivity were found to gradually increase and obtained at an optimum value of $1.0 \mathrm{M}$ sample (Supplementary Fig. 7a). However, the $S_{i}$ and $\sigma_{i}$ decreased when the concentration of NaTFSI was over $2.0 \mathrm{M}$, because higher concentration was easy to form ion clusters and it generated high viscosity which reduced the number of free ions and slowed down the ion diffusion process ${ }^{27,28}$. The thermal conductivity slightly increased with the increasing concentration of NaTFSI and the value ranged from 0.15 to $0.17 \mathrm{~W} \mathrm{~m}^{-1} \mathrm{~K}^{-1}$ (Supplementary Fig. $7 \mathrm{~b}$ ). The dimensionless ionic figure of merit $Z T_{i}$, which is defined as $Z T_{i}=S_{i}^{2} \sigma_{i} T / \lambda_{i}$, is used to characterize the performance of $i$-TE materials ${ }^{29}$. A high thermopower, ionic conductivity and low thermal conductivity of the developed PhNPs greatly contributed to achieving a high $Z T_{i}$. As a result, the $Z T_{i}$ of PhNP-86 with $1.0 \mathrm{M}$ NaTFSI obtained a maximum value of over 0.2 at $298 \mathrm{~K}$, as shown in Fig. 1d and Supplementary Fig. 7b, which is higher than some recently reported $i$-TE materials such as $\mathrm{PEO} / \mathrm{NaOH}(0.014)^{14}$, PSSNa $(0.013)^{30}$ and PVDF-HFP/EMMI:TFSI $(0.007)^{9}$.

Thermoelectric properties analysis. In this work, the weight ratio of NaTFSI/PC is found to have a significant effect on both the thermopower and ionic conductivity of the PhNP. As suggested by a recent work ${ }^{16}$, the generated thermopower can be described by Eq. (2):

$$
S_{t d}=\frac{\sum_{i} q_{i} n_{i}^{0} \hat{S}_{i} D_{i}}{\sum_{i} q_{i}^{2} n_{i}^{0} D_{i}}
$$

where $q, n^{0}, i, \hat{S}$ and $D_{i}$ represent charges, concentration, ion species, the Eastman entropy and diffusion coefficient, respectively. $S_{t d}$ is strongly associated with the change in $\hat{S}_{i}$ and mass transport difference between positive and negative ions at a given temperature difference. Previous work ${ }^{14,16,31}$ suggested that the interaction between ions and polymer matrix was considered to have a substantial impact on the Eastman entropy. To understand the interaction between PVDF-HFP and ions, the Fourier transform infrared spectroscopy (FTIR) characterization from 400 to $950 \mathrm{~cm}^{-1}$ of each PhNPs was conducted, as shown in Fig. 2a. Upon incorporation of NaTFSI/PC into PVDF-HFP, the intensity of the crystalline phase ( $\alpha$-phase) of PVDF-HFP, which is located at $980 \mathrm{~cm}^{-1}\left(-\mathrm{CF}_{2}\right.$ and -CC symmetric stretching), $796 \mathrm{~cm}^{-1}$ (- $\mathrm{CF}_{3}$ stretching), $762 \mathrm{~cm}^{-1}$ ( $-\mathrm{CH}_{2}$ rocking), $614 \mathrm{~cm}^{-1}\left(-\mathrm{CF}_{2}\right.$ bending and CCC skeletal), and $531 \mathrm{~cm}^{-1}\left(-\mathrm{CF}_{2} \text { wagging }\right)^{32,33}$, gradually decreased and peaks of 614 and $762 \mathrm{~cm}^{-1}$ even disappeared at higher concentration of NaTFSI/PC. Meanwhile, the 

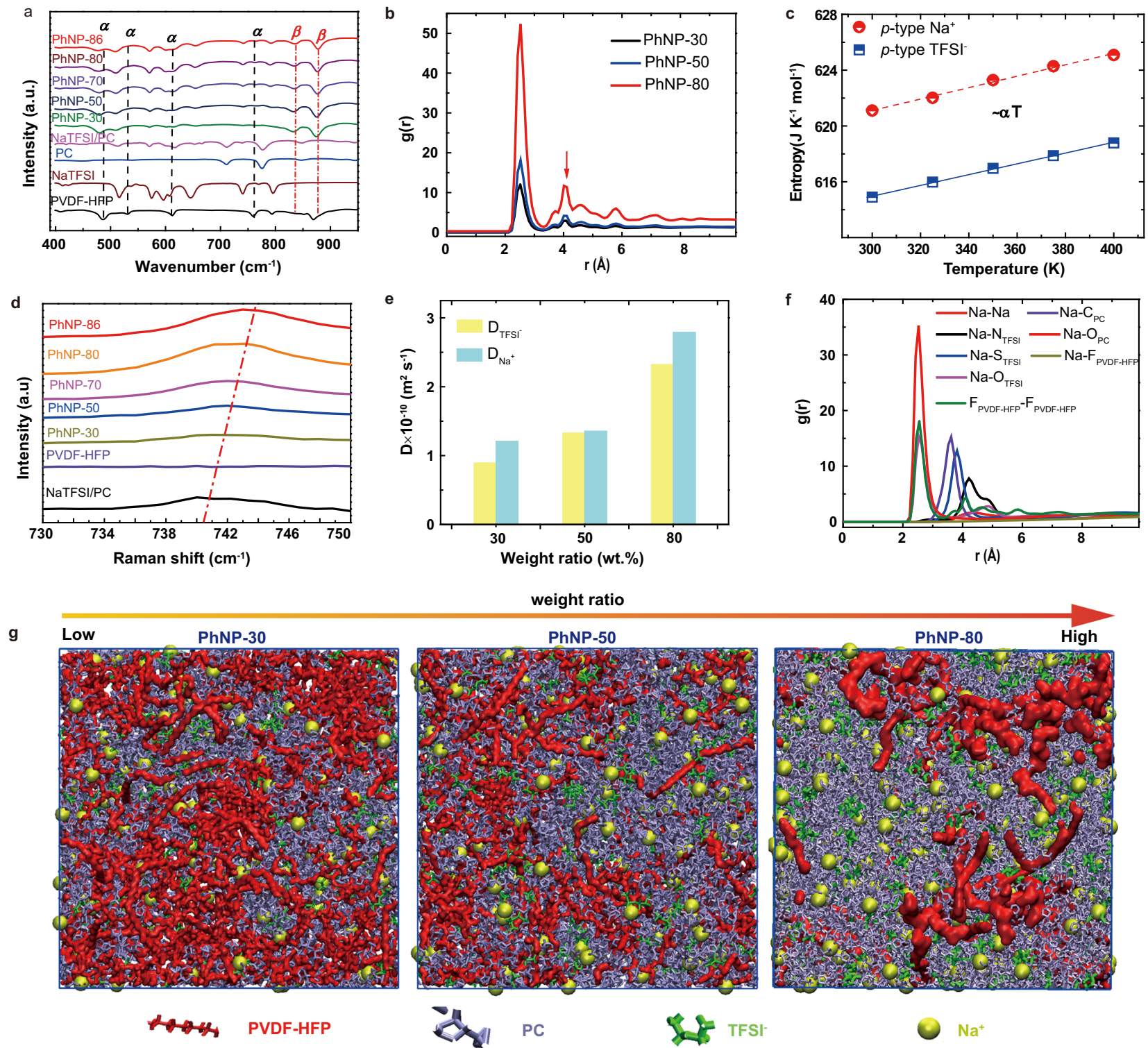

Fig. 2 The structural characterization and atomic-level interaction in PhNPs. a FTIR spectra with a range of $400-950 \mathrm{~cm}^{-1}, \mathbf{b}$ the calculated RDFs of the PhNP-30, -50 and -80 samples, $\mathbf{c}$ the calculated entropy change of cations and anions in PhNP-86, $\mathbf{d}$ the Raman spectra with a range of $733-746 \mathrm{~cm}^{-1}$ of each PhNPs, e the diffusion coefficient of $\mathrm{Na}^{+}$and TFSI- in PhNP-30, -50 and -80 samples, $\mathbf{f}$ the RDF of each interaction among ions and PVDF-HFP of PhNP-50 and $\mathbf{g}$ the snapshots of the PhNP-30, -50 and -80 samples in the MD simulation model.

peak at $879 \mathrm{~cm}^{-1}$ belonging to the amorphous phase ( $\beta$-phase) became dominant and another $\beta$-phase peak at $841 \mathrm{~cm}^{-1}$ appeared. On one hand, as the ion conduction in polymer occurs mainly in the amorphous region ${ }^{21,34}$, the increased amorphous region ( $\beta$-phase) of PVDF-HFP is favored to improve ion mobility. On the other hand, the formation of the $\beta$-phase is attributed to the interaction between the dipole moment of polymer and ions in which a large spontaneous polarization can be generated $^{35,36}$.

Moreover, to gain molecular-level insights into the interactions between the ions and polymer, and the entropy change, we performed all-atom MD simulations and the details of the calculation method are illustrated in Supplementary Information. Figure $2 \mathrm{~g}$ shows the simulation snapshots taken from the PhNP$30,-50$ and -80 samples, where the PVDF-HFP chains, PC small molecules, TFSI ${ }^{-}$anions and $\mathrm{Na}^{+}$cations distribute uniformly in the simulations cell. With the increasing weight ratio of NaTFSI/ PC, the intertwined chains of PVDF-HFP tend to spread and the interactions among chains/ions could be changed accordingly. The scanning electron microscope (SEM) also found that the packed PVDF-HFP was quickly changed to the porous structure of PhNPs and the size of quasi-spherical grains of the polymer chains gradually decreased (Supplementary Fig. 5a-j), which are consistent with the built MD model (Fig. 2g). The calculated radial distribution functions (RDFs) between fluorine $(\mathrm{F})$ atoms in PVDF-HFP showed that the amplitude of the former peak at $2.5 \AA$ increased from the sample PhNP-30 to PhNP-80 (Fig. 2b), indicating more structural heterogeneity in the sample with the increasing weight ratio of NaTFSI/PC. The RDF is defined as the probability of finding a pair of atoms at a particular separation compared to that of a random distribution at the same density. It also suggests that the local density of the PVDF-HFP chains in the PVDF-HFP rich area will be much higher than the average value over the whole simulations and explain the large variation in peak height. Meanwhile, we also observed a peak at $4.2 \AA$ gradually loomed with the increasing weight ratio of NaTFSI/PC. 
This peak strongly implies that partial interaction at $2.5 \AA$ converts to weak force among PVDF-HFP separated chains in PhNP-80, which is consistent with the emerging phase in the experimental finding in Fig. 2a. Furthermore, the entropies of both the $\mathrm{Na}^{+}$cations and $\mathrm{TFSI}^{-}$anions were predicted by the MD simulations ${ }^{37,38}$ (detail in Supplementary Information). They almost exhibited a linear relationship with temperature (Fig. 2c), which is consistent with the second law of thermodynamics. The calculated slope of entropy change of $\mathrm{Na}^{+}$ions $(0.040)$ is larger than that of TFSI $^{-}$ions $(0.038)$ under the same temperature difference, suggesting a larger driving force of $\mathrm{Na}^{+}$ions from entropy change and thus enhancing the thermopower.

Another important factor to improve thermopower is to enlarge mass transport differences of cations and anions which usually enhance potential imbalance. From Raman analysis (Supplementary Fig. 8), it is clear to find the intensity of the peak located at $740-741 \mathrm{~cm}^{-1}$, which is assigned to TFSI $^{-}$anions, gradually becomes more predominant, confirming more amount of NaTFSI/ PC was introduced. Meanwhile, the peak position of TFSI ${ }^{-}$ exhibited a shift to a larger wavelength $\left(743-744 \mathrm{~cm}^{-1}\right)$ direction (Fig. 2d), which indicates that more amount of the TFSI ${ }^{-}$anions formed contact ion pairs ${ }^{28,39}$. The vibrational spectrum of contact ion pairs can be influenced by both ionic interaction and conformational effects. The contact ion pairs tend to form NaTFSI, $\left[\mathrm{Na}(\mathrm{TFSI})_{2}\right]^{-},\left[\mathrm{Na}(\mathrm{TFSI})_{3}\right]^{2-}$ and $\left[\mathrm{Na}(\mathrm{TFSI})_{4}\right]^{3-}$ complexes $^{40}$ as illustrated in Supplementary Fig. 9a. It's speculated that a larger number of TFSI ${ }^{-}$anions is involved in the complexes than $\mathrm{Na}^{+}$ cations which might be responsible for enlarging the mass transport difference between $\mathrm{Na}^{+}$and $\mathrm{TFSI}^{-}$. In addition, the analysis of MD simulation results showed that the diffusion coefficient of $\mathrm{Na}^{+}$ cations $\left(2.79 \times 10^{-10} \mathrm{~m}^{2} \mathrm{~s}^{-1}\right)$ was substantially higher than that of TFSI $^{-}$anions $\left(2.32 \times 10^{-10} \mathrm{~m}^{2} \mathrm{~s}^{-1}\right)$ of PhNP-80 (Fig. 2e) which was consistent with the experimental results by Pulsed-field gradient nuclear magnetic resonance ${ }^{41}$. Moreover, in a negatively charged polymer network, the cations usually show a higher diffusion coefficient according to the experimental ${ }^{16,42}$ and computational analysis ${ }^{43,44}$. As a small portion of cations tend to condensate along the negative sites of polymer chains according to the counterion condensation limiting laws ${ }^{45}$, where these immobilized $\mathrm{Na}^{+}$around the PVDF-HFP chains generate frictional resistance on the free TFSI $^{-}$anions. At the same time, the formed electrostatic field surrounding the PVDF-HFP enables to govern $\mathrm{Na}^{+}$ions transport along the direction from the hot to cold side (Supplementary Fig. 9b). This disparity facilitates the transport of $\mathrm{Na}^{+}$ions and impedes the movement of TFSI ${ }^{-}$ions ${ }^{46}$.

The increased thermal conductivity of PhNPs was ascribed to the enhanced heat transport contribution from electrostatic interactions between ions and the surrounding environment in the system with an increasing weight ratio of NaTFSI/PC. To better understand the interatomic interactions, the RDFs of $\mathrm{Na}-\mathrm{O}_{\mathrm{PC}}, \mathrm{Na}-\mathrm{O}_{\mathrm{TFSI}}, \mathrm{Na}-\mathrm{N}_{\mathrm{TFS}}$, $\mathrm{Na}-\mathrm{S}_{\text {TFSI }}, \mathrm{Na}-\mathrm{C}_{\mathrm{PC}}$, and $\mathrm{F}_{\text {PVDF-HFP }}-\mathrm{F}_{\mathrm{PVDF}}$-HFP pairs of PhNP-50 as a typical example were studied (Fig. 2f). It showed that a peak position at around $2.6 \AA$ between the charged pairs $\left(\mathrm{Na}-\mathrm{Na}, \mathrm{Na}-\mathrm{O}_{\mathrm{PC}}\right.$, and $\left.\mathrm{Na}-\mathrm{O}_{\text {TFSI }}\right)$ which suggested the distance of the charged pairs is more likely to be closer than the other pairs, i. e. Na-F $\mathrm{F}_{\mathrm{PVDF}-\mathrm{HFP}}, \mathrm{Na}-\mathrm{C}_{\mathrm{PC}}$, and $\mathrm{Na}-\mathrm{S}_{\mathrm{TFSI}}$ pairs due to the stronger electrostatic interactions among the former charged pairs. As the weight ratio of NaTFSI/PC increased, they provided larger proportional electrostatic interactions and more thermal pathways for phonon transport due to the transition from weak Van der Waals interaction to strong Coulomb interactions.

Converting $\boldsymbol{p}$-type PhNP to $\boldsymbol{n}$-type. Regulating ion transportation is an effective way to tune the sign of thermopower. Here, tris(pentafluorophenyl)borane (TPFPB) molecule was incorporated to the $p$-type $\mathrm{PhNP}$-86, which could provide a negative-charged enriched environment in the composite. After introducing $1.5 \mathrm{M}$ TPFPB, the generated voltage exhibited a positive value (red line, Fig. 3b) with a $\Delta T(+6 \mathrm{~K})$ at the starting state, which was opposed to the sign of generated voltage of $p$ type PhNP-86. The reversing sign of voltage suggested that a higher concentration of $\mathrm{TFSI}^{-}$ions was accumulated at the cool side (Fig. 3a), indicating TFSI $^{-}$anions dominated the thermodiffusion process, realizing the conversion from $p$-type PhNPs to $n$-type. To comprehensively study the influence of TPFPB on tunning thermopower, a series of TPFPB concentrations from $2.5 \mathrm{mM}$ to $1.5 \mathrm{M}$ were introduced into $p$-type PhNP-86 and assigned as T-PhNP- $x \mathrm{M}$, where $x$ is the concentration of TPFPB. The original porous morphologies gradually became more compact structures, and the pores were even filled at high concentrations of the TPFPB (Supplementary Fig. 10a-c). The energy dispersive X-ray spectroscopy (EDS) mapping of T-PhNP-0.5 M (Supplementary Fig. 10d) demonstrated the distribution of each element of the fabricated $i$-TE materials. Specifically, the boron (B) element only coming from TPFPB was found on the surface of T-PhNPs, confirming that the T-PhNPs composites were successfully formed.

The thermopower of the T-PhNP-2.5 mM and T-PhNP-5 mM decreased to 12.95 and $6.2 \mathrm{mV} \mathrm{K}^{-1}$, respectively, indicating the thermodiffusion of cations was impeded as shown in Fig. 3c. Interestingly, the thermopower of T-PhNPs- $0.01 \mathrm{M}$ is close to zero (Fig. 3d), representing the thermodiffusion of cations is nearly close to anions. Moreover, when the concentration of TPFPB exceeded this critical point, the thermopower became more negative with containing more amount of TPFPB (Fig. 3d, e and Supplementary Fig. 11), strongly suggesting the thermodiffusion of anions was easier than cations of the T-PhNPs. And the maximum value reached around $-6 \pm 1 \mathrm{mV} \mathrm{K}^{-1}$ of T-PhNP-1.5 M, which is comparable with those of the reported organic $n$-type $i$-TE materials so far, such as the PVDF-HFP/EMIM:TFSI $\left(-4 \mathrm{mV} \mathrm{K}^{-1}\right)^{9}$, tetrachloro-perylene bisimide (4Cl-PBI) $\left(-3.02 \mathrm{mV} \mathrm{K}^{-1}\right)^{47}$ at room temperature. From the X-ray photoelectron spectroscopy (XPS) analysis (Supplementary Fig. 12 and Fig. 3f, g), it is clear to find that the intensity of the peak located at $191 \mathrm{eV}$ gradually became stronger (Fig. 3f), which was assigned to $B$ atoms, suggesting more amount of TPFPB was introduced. Moreover, the binding energy of F1s tends to shift from 688 to $686 \mathrm{eV}$ upon adding TPFPB. Since the interaction between $\mathrm{F}$ and $\mathrm{Na}^{+}$could change the energy status of $\mathrm{F}$ of TPFPB, making the binding energy towards the Na-F of which the binding energy is around $684 \mathrm{eV}^{48}$. In addition, the built $\mathrm{MD}$ model of T-PhNP-1.5 M and the calculated RDFs between $\mathrm{Na}^{+}$and atoms of TPFPB molecules from MD simulations are shown in Supplementary Fig. 13a and Fig. 3h, respectively. The RDFs indicate that the F atom in TPFPB molecules has the strongest interaction with $\mathrm{Na}^{+}$ions. It's speculated that the incorporation of TPFPB molecules not only traps $\mathrm{Na}^{+}$ions but also breaks up the interactions between $\mathrm{Na}^{+}$and PVDF-HFP and disturbs the transport path of $\mathrm{Na}^{+}$ions along the PVDF-HFP polymer chain. Besides, MD results suggested that the slope of entropy change with temperature of TFSI ${ }^{-}$anions (0.0365) is larger than that of $\mathrm{Na}^{+}$ cations (0.0317) in $n$-type T-PhNP-1.5 M (Supplementary Fig. 13b, c). It indicates that the addition of TPFPB makes a larger change in entropy and creates larger driving force for TFSI ${ }^{-}$anions than $\mathrm{Na}^{+}$ cations, which is opposite to that of $p$-type PhNPs, realizing the transition from $p$-type to $n$-type of the developed $i$-TE materials. Furthermore, the measured ionic conductivities of $n$-type T-PhNP$0.5 \mathrm{M}$ and $-1.0 \mathrm{M}$ were $1.5 \times 10^{-3} \mathrm{~S} \mathrm{~cm}^{-1}$ and $9.33 \times 10^{-4} \mathrm{~S} \mathrm{~cm}^{-1}$, respectively, (Supplementary Fig. 14). The introduced TPFPB not only trapped parts of $\mathrm{Na}^{+}$ions, but also the formed dense structure may improve crystallinity, reducing the number and mobility of ions. The thermal conductivities of T-PhNP-0.5 M and $-1.0 \mathrm{M}$ at 

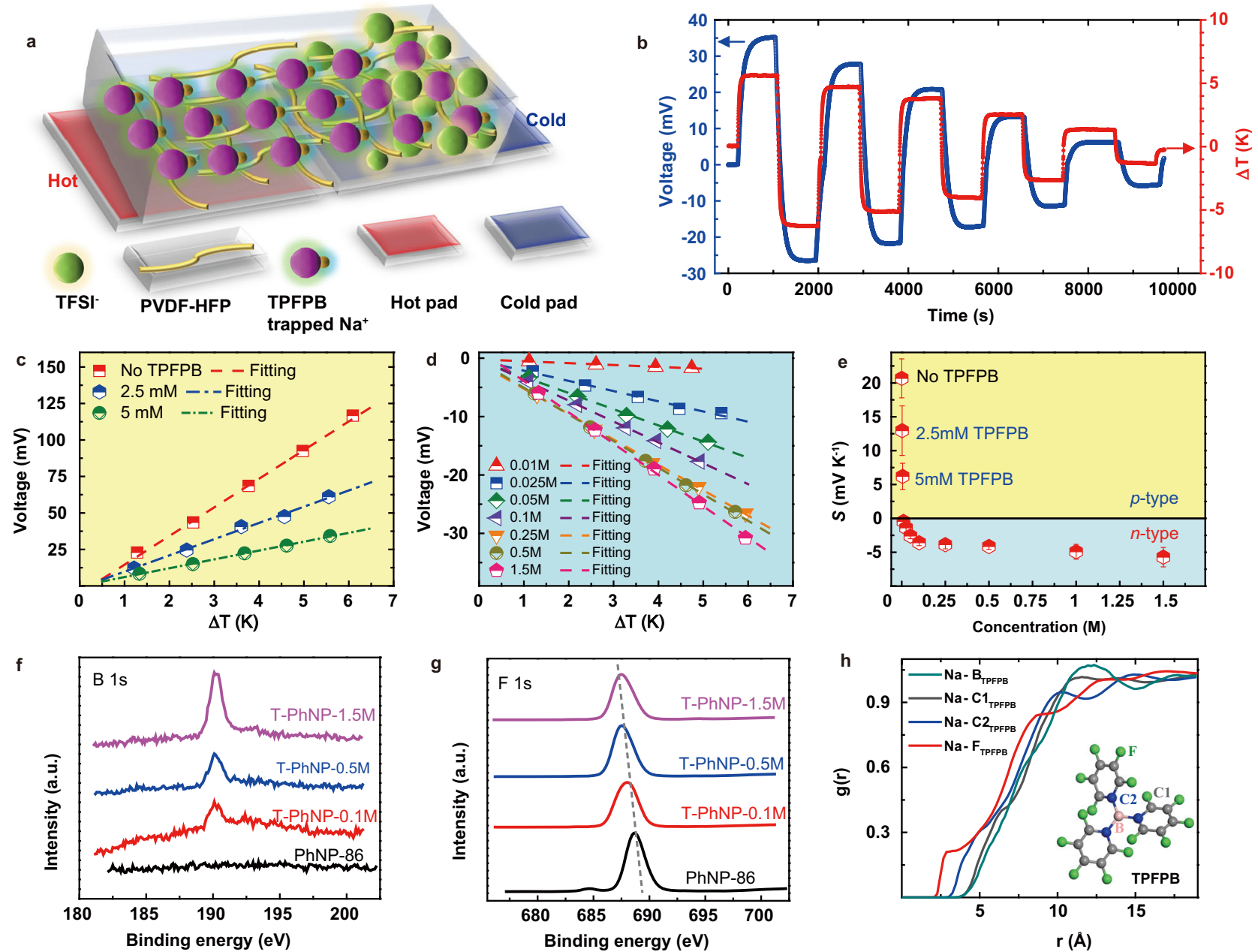

Fig. 3 The conversion from $\boldsymbol{p}$-type PhNP to $\boldsymbol{n}$-type T-PhNP. a The schematic of ion transport of $n$-type (TFSI- anions dominate thermodiffusion) T-PhNP $i$-TE materials. $\mathbf{b}$ The generated voltage under a series of temperature differences of T-PhNP-1.5 M. c, d The plot of fitting curves of $\Delta V_{i}-\Delta T$ and $\mathbf{e}$ the calculated thermopower of each T-PhNPs. XPS characterization of $\mathbf{f} B(1 \mathrm{~s})$ and $\mathbf{g} \mathrm{F}(1 \mathrm{~s})$ peak of T-PhNPs. $\mathbf{h}$ The radial distribution function of Na+ cations and the atoms of TPFPB molecules.

$298 \mathrm{~K}$ are $0.165 \mathrm{~W} \mathrm{~m}^{-1} \mathrm{~K}^{-1}$ and $0.185 \mathrm{~W} \mathrm{~m}^{-1} \mathrm{~K}^{-1}$, respectively, gradually increasing after introducing TPFPB. The figure of merit of $Z T_{i}$ for $n$-type T-PhNP-0.5 M and $-1.0 \mathrm{M}$ are 0.0046 and 0.0037 , respectively.

For practical application, the performance of both $p$ - and $n$-type $i$-TE materials under different $\mathrm{RH}$ was studied. The ionic thermopower of the $p$-type PhNP-86 sample increased from $8.12 \mathrm{mV} \mathrm{K}^{-1}$ ( $40 \% \mathrm{RH})$ to $26.62 \mathrm{mV} \mathrm{K}^{-1}$ (90\% RH) as shown in Supplementary Fig. 15a. As the porous structure of PhNP-86 and the hydrophilic nature of sodium salt tend to absorb water from the moisture environment, the absorbed water could fill the space in the polymer matrix $^{49,50}$ and help improve the dissociation of the NaTFSI counter-ions by weakening the electrostatic potential. However, the thermopower of the $n$-type T-PhNP-1.0 M exhibited a decreasing tendency with the increasing humidity (Supplementary Fig. 15b). It's suspected that the absorbed water may interact with TPFPB at high humidity ${ }^{51,52}$, which may block the ions transportation pathway and reduce ions' mobility. In addition, the durability of the $n$-type T-PhNP-1.0 M sample was further investigated. It's clear to find the thermopower of T-TPFPB-1.0 M remained at an almost constant value $\left(-5\right.$ to $\left.-6 \mathrm{mV} \mathrm{K}^{-1}\right)$ for over more than 18 days at $68 \%$ RH, demonstrating strong durability (Supplementary Fig. 15c and d). Moreover, it's crucial to deposit a uniform metal contact layer on $i$-TE materials when fabricating $i$-TE modules. But it's almost impossible to deposit a uniform metal contact layer on the surface of liquids or gels under a high vacuum condition ${ }^{53}$ Interestingly, our developed all-solid-state $n$-type T-PhNP can easily achieve the uniform deposition of an Au metal layer by the physical vacuum evaporation (PVD) method attributed to the solid physical features, as shown in Supplementary Fig. 16a, c. For comparison, the reported PVDF-HFP/EMIM:TFSI gel ${ }^{9}$ was selected through the same PVD process. Unluckily, it formed a discontinuous Au layer and the color of the surface became black, representing such liquid-dominated gels cannot survive when involved in the high vacuum process (Supplementary Fig. 16b, d). Clearly, it is a significant step to prove that the possibility of $i$-TE materials can be further extended to develop micro/nano ionic TEbased devices by standard fabrication process in the semiconductor industry.

The prototype of all-solid-state $i$-TE devices. A planar $i$-TEG was fabricated, which included 13 pairs of $p$-type PhNP-86 and $n$ type T-PhNP-1.5 M films shown in Fig. 4a. The produced thermal voltage of the $i$-TEG reached over $\sim 2.6 \mathrm{~V}$ at $\Delta T=10 \mathrm{~K}$ in about $200 \mathrm{~s}$ (Fig. 4e) in the air, indicating good thermal response property. The overall thermopower of this $i$-TEG reached as high as $0.265 \mathrm{~V} \mathrm{~K}^{-1}$, which is near to the theoretical sum of the thermopower of PhNP-86 and T-PhNP-1.5 M TE legs $\left(0.338 \mathrm{~V} \mathrm{~K}^{-1}\right)$ (Supplementary Fig. 17). To the best of our knowledge, the 
a

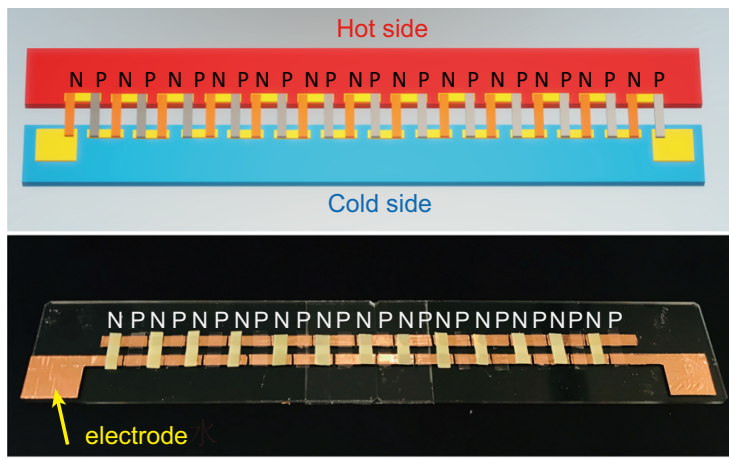

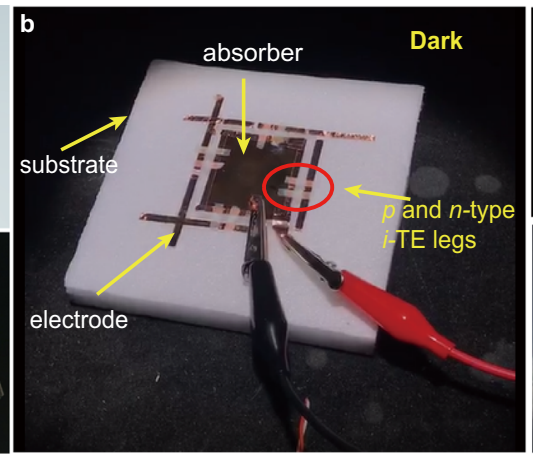

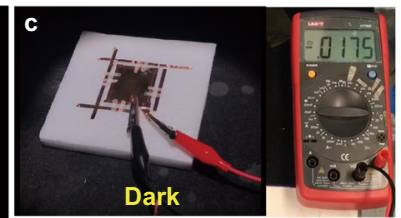

d

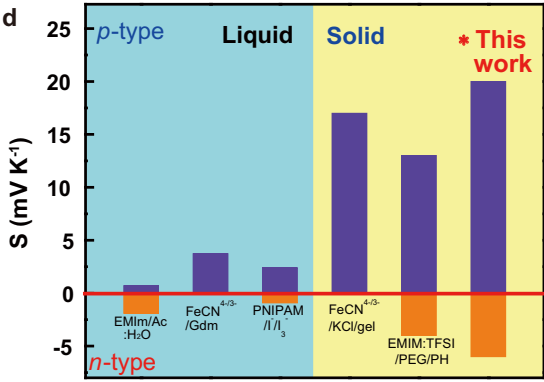

i-TE materials
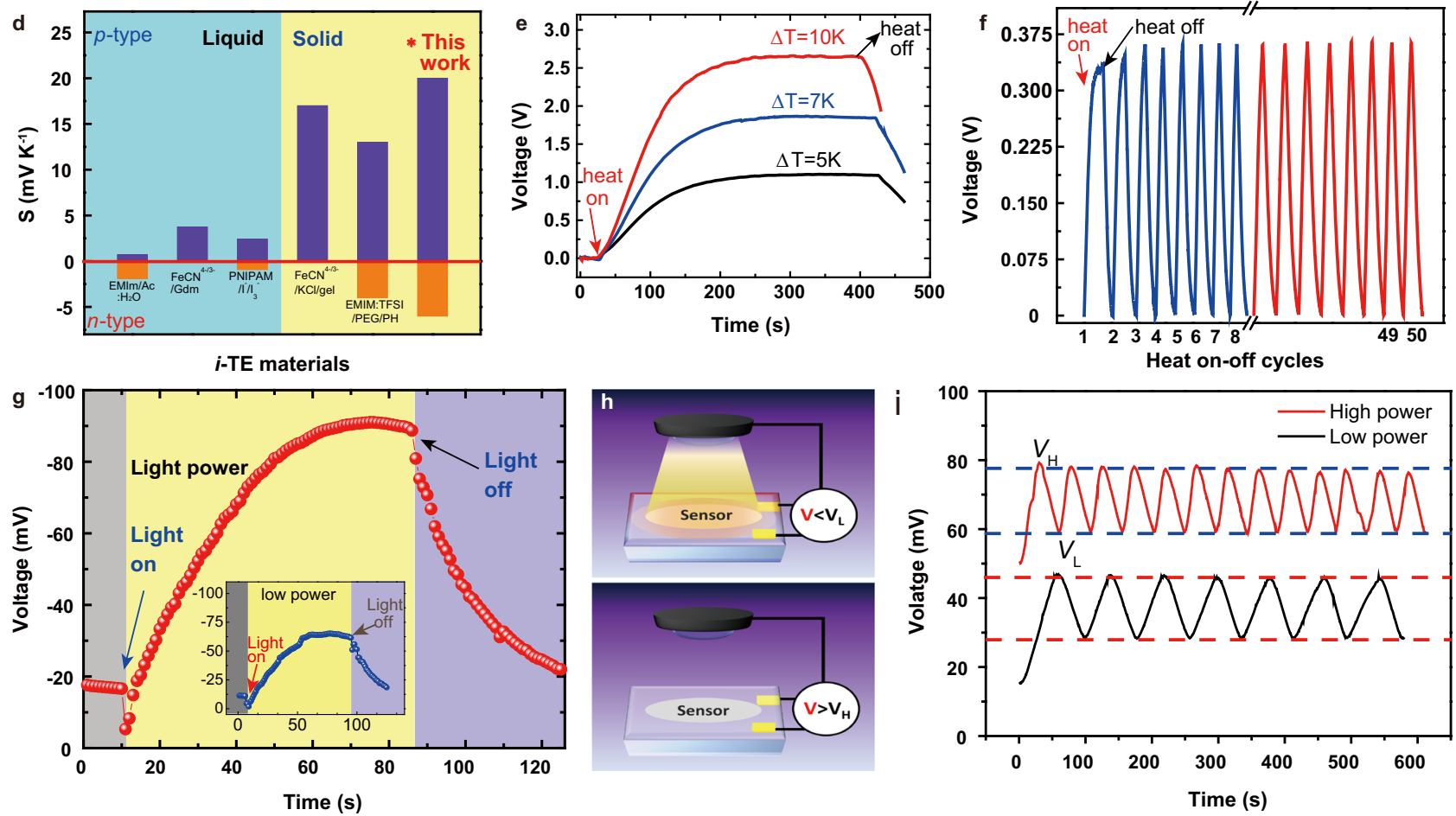

Fig. 4 The performance of the prototype of all-solid-state $\boldsymbol{i}$-TE devices. a The digital photo of the design and the fabricated in-plane $i$-TEG with 13 pairs of $p$ - $n$ thermocouples. b, c The demonstration of ionic-thermoelectric thermal sensors. $\mathbf{d}$ Comparison of the thermopower range for the recently reported $i$-TE materials realizing $p-n$ conversion $9,17,18$. e The thermoelectric voltage $(V)$ vs time $(t)$ curves of the fabricated $i$-TEG under various $\Delta T$. $\mathbf{f}$ The 50 -cycles heat

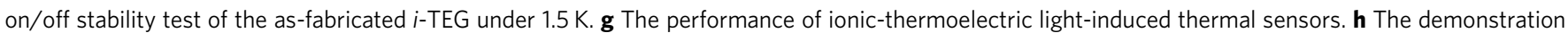
and (i) performance of a self-powered LiTS system that can automatically control the lamp.

developed PhNP/T-PhNP thermocouple demonstrated a wide thermopower tuned range compared to the recently reported $i$-TE thermocouples up to the present, as summarized in Fig. $4 \mathrm{~d}$. Moreover, after repeating the heating-cooling test in the air, the produced thermal voltage of the fabricated $i$-TEG demonstrated high repeatability after 50 cycles (Fig. $4 \mathrm{f}$ ). Furthermore, to evaluate the generated power of the developed $i$-TE materials, an ionic thermoelectric capacitor ( $i$-TEC) was built to convert ion charges originating from the heat to power an external load through 4 steps in one cycle as shown in Supplementary Fig. 18a. Briefly, a thermal voltage $\left(V_{1}\right)$ of a single PhNP film was generated at $\Delta T=1.5 \mathrm{~K}$. After connecting an external resistor, a pulse-like voltage $\left(V_{2}\right)$ of the external resistor was produced, which was caused by the accumulation of electrons and holes of the electrodes to balance $V_{1}$. Then, the $\Delta T$ was removed and the external resistor was disconnected simultaneously, the $V_{1}$ turned negative because the $\mathrm{Na}^{+}$and TFSI ${ }^{-}$ recovered to the original status while the electrons and holes stayed at the two electrodes. The last step was to power the resistor by connecting to the $i$-TEC again (Supplementary Fig. 18b). The output energy was calculated according to $\mathrm{E}=\int V^{2} / R d t$, where $V$ and $R$ are the voltage and the resistance of the external load, respectively ${ }^{54}$. The calculated power and energy of $i$-TEC was $2.08 \mu \mathrm{W} \mathrm{cm} \mathrm{cm}^{-2}$ and $833.1 \mu \mathrm{J} \mathrm{cm}^{-2}$ when a resistor with $1 \mathrm{k} \Omega$ was connected for the time duration $400 \mathrm{~s}$ of the last stage (Supplementary Fig. 18c, d), which is comparable to the recent works, such as $2.43 \mu \mathrm{W} \mathrm{m} \mathrm{m}^{-2}$ and $714 \mu \mathrm{J} \mathrm{m}^{-2}$ of polyaniline (PANI)/poly(2acrylamido-2-methyl-1-propane sulfonic acid) (PAAMPSA)/(phytic acid) (PA) with a resistor $6250 \Omega^{10}, 0.83 \mu \mathrm{W} \mathrm{m}^{-2}$ and $223.8 \mu \mathrm{J} \mathrm{m}^{-2}$ of PVDF-HFP/1-ethyl-3-methylimidazolium dicyanamide (EMIM:DCA) with a resistor of $5 \mathrm{k} \Omega^{8}$.

Light-induced thermal sensors. We further presented a selfpowered and highly sensitive TS to detect light-induced heat by taking the unique advantages of the $i$-TE materials. The lightinduced thermal sensor (LiTS) is fabricated with 4 pairs of PhNP and T-PhNP, where the $i$-TE legs are connected in series and suspended between the titanium nitride $(\mathrm{TiN})^{55}$ absorber and the substrate (Fig. 4b). The TiN absorber was to generate heat under light illumination and the substrate was used as a heat sink. The LiTS could exhibit a huge advantage of high-temperature 
sensitivity over the commercial K-type thermocouple ${ }^{56}$ with a temperature sensitivity of $0.04 \mathrm{mV} \mathrm{K}^{-1}$. When the lamp is turned on, the voltage was suddenly generated by the LiTS and varied from -17.5 to $-90 \mathrm{mV}$ within the $50 \mathrm{~s}$ (Fig. 4c, g). Once the illumination is turned off, it can be observed the voltage dropped rapidly to the original status. After reducing the illumination power, the generated peak voltage decreased to $-56 \mathrm{mV}$, which proved the sensing signal can be changed according to the variation of illuminance power correspondingly (Fig. $4 \mathrm{~g}$ inset blue curve). Moreover, the sensor has been developed into a selfpowered system that can automatically control the lamp without the need for an external amplifier circuit. As shown in Fig. 4h, once the light was on, the voltage was immediately generated of the system. When the voltage exceeded the upper limit, the system would automatically turn off the light. Then the temperature difference gradually decreased, and the voltage would drop accordingly. Until the lower limit was reached, the system would turn on the lights again and the voltage raised again. By changing the lighting power, the system can automatically control the on/ off frequency of the lamp, where the greater lighting power has a faster response (Fig. 4i). Clearly, the developed self-powered system has the characteristics of high sensitivity, repeatability, and good consistency. Since the limiting factor for the speed of the $i$-TE LiTS is the bulk TE component, further miniaturization of the basic TE voltage generating unit will allow much shorter response times, which will be demonstrated in our future work. Overall, the $i$-TE based system provides higher voltage signals for the same thermal stimulus, which not only improves the resolution of the detector but also benefits from absolute large changes in voltage, especially for the heat-gated transistors, heat mapping 56,57 .

\section{Discussion}

This work successfully developed all-solid-state PVDF-HFP/ NaTFSI/PC with high thermopower $\left(S_{i}\right)$ of $+20 \mathrm{mV} \mathrm{K}^{-1}$ attributed to the enhanced ions/polymer interactions and mass transport difference. Meanwhile, the $p$ - $n$ conversion in all-solid-state $\mathrm{PhNP}$ from +20 to $-6 \mathrm{mV} \mathrm{K}^{-1}$ was achieved by incorporating TPFPB to impede the transportation of $\mathrm{Na}^{+}$ions. Meanwhile, the mechanism of $p-n$ conversion was studied in detail based on the analysis of Eastman entropy changes in all-solid-state PhNP $i$-TE polymers material at the atomic scale. In addition, this work also systematically investigated and optimized the TE performance of both $p$ - and $n$-type materials with various compositions under different conditions. Moreover, an all-solid-state $i$-TE generator with 13 pairs of $p-n$ legs generated a high voltage over $2.6 \mathrm{~V}$ $(\Delta T=10 \mathrm{~K})$. Besides, a self-powered TS demonstrated high sensitivity and good consistency. This work obtains high thermopower and realizes the $p-n$ conversion in the all-solid-state $i$-TE polymer material simultaneously, which is beneficial for the development of next-generation high-performance flexible $i$-TE systems.

\footnotetext{
Methods

MD calculation of thermal conductivity and ionic diffusion of PhNP systems. All-atom MD simulations were performed to predict the thermal conductivity and ionic diffusion properties of the solid-state $i$-TE material PVDF-HFP/NaTFSI/PC. The initial structures of PVDF-HFP chains, NaTFSI, and PC molecules were generated using the freely available AVOGADRO software package ${ }^{58}$. These structures were then geometrically optimized via the use of Generalized Amber Force field (GAFF) ${ }^{26}$. The structures were considered to be geometrically optimized when the energy difference between two successive iterations dropped below a threshold value $\left(10^{-8} \mathrm{~kJ} \mathrm{~mol}^{-1}\right)$. The LigParGen web-based service ${ }^{59}$ is used to obtain the OPLS force-field parameters (Supplementary Fig. 19 and Supplementary Table 1) and partial atomic charges for the PVDF-HFP, NaTFSI, and PC molecules, which are placed together in a cubic simulation box with a dimension of $25 \times 25 \times 25 \mathrm{~nm}^{3}$ using PACKMOL ${ }^{60}$. We adjusted the number of PVDF-HFP, NaTFSI, and PC molecules so that the size of each system was kept consistent.
}

Periodic boundary conditions in all three dimensions were implemented. The cutoff distance for long-range energy calculations was set to be $12 \AA$. The contribution of long-range interactions was calculated via the particle-particle-particle-mesh solver ${ }^{61}$. The Newton's equations of motion were time-integrated with a time-step of $1 \mathrm{fs}$ using Large-scale Atomic/Molecular Massively Parallel Simulation package ${ }^{62}$ developed by Sandia National Laboratories. The Visual Molecular Dynamics ${ }^{63}$ was used to visualize the trajectories generated during MD simulations. Each sample was equilibrated via the use of NPT simulations at $294 \mathrm{~K}$ and $1 \mathrm{~atm}$ over a period of $2 \mathrm{~ns}$. Following this, a further $5 \mathrm{~ns}$ simulation was performed in the NVT ensemble. We recorded a trajectory of 1000 frames that were generated every 1 ps. The whole trajectory was then used for calculating the RDFs and mean square displacement curves (Supplementary Fig. 20). In thermal transport simulations, the heat source and heat sink were set as 320 and $280 \mathrm{~K}$, respectively, using Langevin thermostats (Supplementary Fig. 21a). The system runs in the NVE ensemble for $1.5 \mathrm{~ns}$ to record heat flux and temperature gradient across the system (Supplementary Fig. $21 \mathrm{~b}, \mathrm{c}$ ). The thermal conductivity of the PhNP-30, -50 , and -80 samples was calculated based on Fourier law using non-equilibrium molecular dynamics simulation ${ }^{64}$

\section{Data availability}

The authors declare that the main data supporting the findings of this study are available within the article and its Supplementary Information files. Extra data are available from the corresponding author upon reasonable request (maweigang@tsinghua.edu.cn).

\section{Code availability}

The code of the entropy calculation is available at https://github.com/eastsheng/entropy.

Received: 17 December 2020; Accepted: 17 December 2021; Published online: 11 January 2022

\section{References}

1. Yokota, T. et al. Ultraflexible, large-area, physiological temperature sensors for multipoint measurements. Proc. Natl Acad. Sci. USA. 112, 14533-14538 (2015)

2. Shi, C. et al. Application of a sub-0.1-mm(3) implantable mote for in vivo realtime wireless temperature sensing. Sci. Adv. 7, eabf6312 (2021).

3. Zhao, L. D. et al. Ultralow thermal conductivity and high thermoelectric figure of merit in SnSe crystals. Nature 508, 373-377 (2014).

4. Russ, B., Glaudell, A., Urban, J. J., Chabinyc, M. L. \& Segalman, R. A. Organic thermoelectric materials for energy harvesting and temperature control. Nat. Rev. Mater. 1, 16050 (2016).

5. Zhao, L. D. et al. Ultrahigh power factor and thermoelectric performance in hole-doped single-crystal SnSe. Science 351, 141-144 (2016).

6. Snyder, G. J. \& Toberer, E. S. Complex thermoelectric materials. Nat. Mater. 7, 105-114 (2008).

7. Poudel, B. et al. High-thermoelectric performance of nanostructured bismuth antimony telluride bulk alloys. Science 320, 634-638 (2008).

8. Cheng, H. L., He, X., Fan, Z. \& Ouyang, J. Y. Flexible quasi-solid state ionogels with remarkable seebeck coefficient and high thermoelectric properties. Adv. Energy Mater. 9, 1901085 (2019).

9. Zhao, D. et al. Polymer gels with tunable ionic Seebeck coefficient for ultrasensitive printed thermopiles. Nat. Commun. 10, 1093 (2019).

10. Akbar, Z. A., Jeon, J. W. \& Jang, S. Y. Intrinsically self-healable, stretchable thermoelectric materials with a large ionic Seebeck effect. Energy Environ. Sci. 13, 2915-2923 (2020).

11. Li, S. \& Zhang, Q. Ionic gelatin thermoelectric generators. Joule 4, 1628-1629 (2020).

12. Duan, J. J. et al. Liquid-state thermocells: opportunities and challenges for low-grade heat harvesting. Joule 5, 768-779 (2021).

13. Yang, B. X. \& Portale, G. Ionic thermoelectric materials for waste heat harvesting. Colloid Polym. Sci. 299, 465-479 (2021)

14. Zhao, D. et al. Ionic thermoelectric supercapacitors. Energy Environ. Sci. 9, 1450-1457 (2016)

15. Jiao, F. et al. Ionic thermoelectric paper. J. Mater. Chem. A 5, 16883-16888 (2017)

16. Han, C. G. et al. Giant thermopower of ionic gelatin near room temperature. Science 368, 1091 (2020).

17. Jia, H., Ju, Z., Tao, X., Yao, X. \& Wang, Y. P-N conversion in a water-ionic liquid binary system for nonredox thermocapacitive converters. Langmuir 33, 7600-7605 (2017).

18. Duan, J. J. et al. P-N conversion in thermogalvanic cells induced by thermosensitive nanogels for body heat harvesting. Nano Energy 57, 473-479 (2019).

19. Kim, B., Hwang, J. U. \& Kim, E. Chloride transport in conductive polymer films for an n-type thermoelectric platform. Energy Environ. Sci. 13, 859-867 (2020) 
20. Yue, L. P. et al. All solid-state polymer electrolytes for high-performance lithium ion batteries. Energy Storage Mater. 5, 139-164 (2016).

21. Zhou, D., Shanmukaraj, D., Tkacheva, A., Armand, M. \& Wang, G. X. Polymer electrolytes for lithium-based batteries: advances and prospects. Chem 5, 2326-2352 (2019).

22. Chi, C. et al. Silicon-nanoforest-based solvent-free micro-supercapacitor with ultrahigh spatial resolution via ic-compatible in-situ fabrication for on-chip energy storage. J. Mater. Chem. A 8, 22736-22744 (2020).

23. Yeon, S. H., Kim, K. S., Choi, S., Cha, J. H. \& Lee, H. Characterization of PVdF(HFP) gel electrolytes based on 1-(2-Hydroxyethyl)-3-methyl imidazolium ionic liquids. J. Phys. Chem. B 109, 17928-17935 (2005).

24. Bai, J. J., Lu, H. M., Cao, Y., Li, X. D. \& Wang, J. R. A novel ionic liquid polymer electrolyte for quasisolid state lithium air batteries. RSC Adv. 7, 30603 (2017).

25. Zhou, W. X. et al. Thermal conductivity of amorphous materials. Adv. Funct. Mater. 30, 1903829 (2020).

26. An, M. et al. Predictions of thermo-mechanical properties of cross-linked polyacrylamide hydrogels using molecular simulations. Adv. Theory Simul. 2, 1800153 (2019).

27. Flores, E., Avall, G., Jeschke, S. \& Johansson, P. Solvation structure in dilute to highly concentrated electrolytes for lithium-ion and sodium-ion batteries. Electrochim. Acta 233, 134-141 (2017).

28. Geng, C. X. et al. Influence of salt concentration on the properties of sodiumbased electrolytes. Small Methods 3, 1800208 (2019).

29. Wang, H. et al. Ionic thermoelectric figure of merit for charging of supercapacitors. Adv. Electron. Mater. 3, 1700013 (2017).

30. Ail, U. et al. Thermoelectric properties of polymeric mixed conductors. $A d v$. Funct. Mater. 26, 6288-6296 (2016).

31. Bonetti, M., Nakamae, S., Roger, M. \& Guenoun, P. Huge Seebeck coefficients in nonaqueous electrolytes. J. Chem. Phys. 134, 114513 (2011).

32. Das, S. \& Ghosh, A. Charge carrier relaxation in different plasticized PEO/ PVDF-HFP blend solid polymer electrolytes. J. Phys. Chem. B 121, 5422-5432 (2017).

33. Rahaman, M. H. A. et al. Effect of gamma irradiation on poly(vinyledene difluoride)-lithium bis(oxalato) borate electrolyte. Phys. Chem. Chem. Phys. 16, 11527-11537 (2014).

34. Boaretto, N., Meabe, L., Martinez-Ibanez, M., Armand, M. \& Zhang, H. Review-polymer electrolytes for rechargeable batteries: from nanocomposite to nanohybrid. J. Electrochem. Soc. 167, 070524 (2020).

35. Lee, S. G., Ha, J. W., Sohn, E. H., Park, I. J. \& Lee, S. B. Enhancement of polar crystalline phase formation in transparent PVDF-CaF2 composite films. Appl. Surf. Sci. 390, 339-345 (2016).

36. Ruan, L. X. et al. Properties and applications of the $\beta$ phase poly(vinylidene fluoride). Polymers 10, 228 (2018).

37. Fleck, M. \& Zagrovic, B. Configurational entropy components and their contribution to biomolecular complex formation. J. Chem. Theory Comput. 15, 3844-3853 (2019).

38. Wei, Y. Y. et al. A molecular dynamics study combining with entropy calculation on the packing state of hydrophobic chains in micelle interior. $J$. Mol. Liq. 283, 860-866 (2019).

39. Boschin, A. \& Johansson, P. Characterization of NaX (X: TFSI, FSI) - PEO based solid polymer electrolytes for sodium batteries. Electrochim. Acta 175, 124-133 (2015).

40. Monti, D., Jonsson, E., Palacin, M. R. \& Johansson, P. Ionic liquid based electrolytes for sodium-ion batteries: $\mathrm{Na}^{+}$solvation and ionic conductivity. $J$. Power Sources 245, 630-636 (2014).

41. Konefal, R. et al. Effect of PAMAM dendrimers on interactions and transport of LiTFSI and NaTFSI in propylene carbonate-based electrolytes. Polymers 12, 1595 (2020).

42. Kamcev, J., Paul, D. R., Manning, G. S. \& Freeman, B. D. Ion diffusion coefficients in ion exchange membranes: significance of counterion condensation. Macromolecules 51, 5519-5529 (2018).

43. Aryal, D. \& Ganesan, V. Reversal of salt concentration dependencies of salt and water diffusivities in polymer electrolyte membranes. ACS Macro Lett. 7, 739-744 (2018).

44. Aryal, D. \& Ganesan, V. Impact of cross-linking of polymers on transport of salt and water in polyelectrolyte membranes: a mesoscopic simulation study. J. Chem. Phys. 149, 224902 (2018).

45. Manning, G. S. Limiting laws and counterion condensation in polyelectrolyte solutions. 8. Mixtures of counterions, species selectivity, and valence selectivity. J. Phys. Chem. 88, 6654-6661 (1984).

46. $\mathrm{Li}, \mathrm{T}$. et al. Cellulose ionic conductors with high differential thermal voltage for low-grade heat harvesting. Nat. Mater. 18, 608-613 (2019).

47. Jiang, Q. L. et al. High thermoelectric performance in n-type perylene bisimide induced by the Soret effect. Adv. Mater. 32, 2002752 (2020).

48. Fondard, J. et al. SEI composition on hard carbon in Na-ion batteries after long cycling: influence of salts (NaPF6, NaTFSI) and additives (FEC, DMCF). J. Electrochem. Soc. 167, 070526 (2020).
49. Wang, H., Ail, U., Gabrielsson, R., Berggren, M. \& Crispin, X. Ionic seebeck effect in conducting polymers. Adv. Energy Mater. 5, 1500044 (2015).

50. Femandes, L. C., Correia, D. M., Pereira, N., Tubio, C. R. \& Lanceros-Mendez, S. Highly sensitive humidity sensor based on ionic liquid-polymer composites. ACS Appl. Polym. Mater. 1, 2723-2730 (2019).

51. Yurash, B. et al. Towards understanding the doping mechanism of organic semiconductors by Lewis acids. Nat. Mater. 18, 1327-1334 (2019).

52. Suh, E. H. et al. Bronsted acid doping of P3HT with largely soluble tris(pentafluorophenyl)borane for highly conductive and stable organic thermoelectrics via one-step solution mixing. Adv. Energy Mater. 10, 2002521 (2020).

53. Kyeremateng, N. A., Brousse, T. \& Pech, D. Microsupercapacitors as miniaturized energy-storage components for on-chip electronics. Nat. Nanotechnol. 12, 7-15 (2017).

54. Fang, Y. L. et al. Stretchable and transparent ionogels with high thermoelectric properties. Adv. Funct. Mater. 30, 2004699 (2020)

55. Li, Y. et al. Solution-processed all-ceramic plasmonic metamaterials for efficient solar-thermal conversion over $100-727^{\circ} \mathrm{C}$. Adv. Mater. 33, 2005074 (2021).

56. Chaharsoughi, M. S. et al. Ultrasensitive electrolyte-assisted temperature sensor. npj Flex. Electron. 4, 23 (2020).

57. Zhao, D., Fabiano, S., Berggren, M. \& Crispin, X. Ionic thermoelectric gating organic transistors. Nat. Commun. 8, 14214 (2017).

58. Hanwell, M. D. et al. Avogadro: an advanced semantic chemical editor, visualization, and analysis platform. J. Cheminformatics 4, 17 (2012)

59. Dodda, L. S., de Vaca, I. C., Tirado-Rives, J. \& Jorgensen, W. L. LigParGen web server: an automatic OPLS-AA parameter generator for organic ligands. Nucleic Acids Res 45, W331-W336 (2017).

60. Martinez, L., Andrade, R., Birgin, E. G. \& Martinez, J. M. PACKMOL: a package for building initial configurations for molecular dynamics simulations. J. Comput. Chem. 30, 2157-2164 (2009).

61. Demir, B., Chan, K. Y. \& Searles, D. J. Structural electrolytes based on epoxy resins and ionic liquids: a molecular-level investigation. Macromolecules 53, 7635-7649 (2020)

62. Plimpton, S. Fast parallel algorithms for short-range molecular-dynamics. J. Comput. Phys. 117, 1-19 (1995).

63. Humphrey, W., Dalke, A. \& Schulten, K. VMD: visual molecular dynamics. J. Mol. Graph. Model. 14, 33-38 (1996).

64. An, M. et al. Mass difference and polarization lead to low thermal conductivity of graphene-like carbon nitride (C3N). Carbon 162, 202-208 (2020).

\section{Acknowledgements}

The authors are thankful for the financial support by the National Natural Science Foundation of China (Grant No. 52106095, 52176078, 52006130, 51636002 and 51827807), Tsinghua University Initiative Scientific Research Program, China Postdoctoral Science Foundation (Grant No. 2020M680024, 2020M670321, 2021T140359, and 2021T140360), Tsinghua-Foshan Innovation Special Fund (2019THFS0125).

\section{Author contributions}

C.C., M.A., W.G.M., and B.L.H. conceived and designed the project. W.G.M., B.L.H., and X.Z. supervised this project. C.C. fabricated and tested the ionic thermoelectric materials and designed the experiment work. M.A., H.D., and B.D. contributed to the MD simulation. X.Q. and G.L. performed the ionic Seebeck measurement. Y.W., R.Z, and H.H. performed the FTIR and Raman test and discussion. Y.L. and C.J. contributed to the SEM characterization. C.C., M.A., and W.G.M. wrote the paper. All authors contributed to discussion and manuscript preparation.

\section{Competing interests}

The authors declare no competing interests.

\section{Additional information}

Supplementary information The online version contains supplementary material available at https://doi.org/10.1038/s41467-021-27885-2.

Correspondence and requests for materials should be addressed to Weigang Ma or Baoling Huang.

Peer review information Nature Communications thanks Jianyong Ouyang and the other anonymous reviewer(s) for their contribution to the peer review this work

Reprints and permission information is available at http://www.nature.com/reprints

Publisher's note Springer Nature remains neutral with regard to jurisdictional claims in published maps and institutional affiliations. 
(c) (i) Open Access This article is licensed under a Creative Commons Attribution 4.0 International License, which permits use, sharing, adaptation, distribution and reproduction in any medium or format, as long as you give appropriate credit to the original author(s) and the source, provide a link to the Creative Commons license, and indicate if changes were made. The images or other third party material in this article are included in the article's Creative Commons license, unless indicated otherwise in a credit line to the material. If material is not included in the article's Creative Commons license and your intended use is not permitted by statutory regulation or exceeds the permitted use, you will need to obtain permission directly from the copyright holder. To view a copy of this license, visit http://creativecommons.org/ licenses/by/4.0/.

(C) The Author(s) 2022 